\title{
An Anti-Collision Algorithm based on Slot Location and Tag Estimation
}

\author{
Yong Liu,Xingzhong Xiong \\ Sichuan University of Science \& Engineering \\ E-mail:1ytj200379@126.com
}

\begin{abstract}
RFID is one of the most important technologies in the Internet of Things (IoT), which has been widely used in all kinds of fields. However, a key problem is the tag collision when multiple tags reflect simultaneously to a reader. In traditional algorithm, slot location is always ignored. We present an anti-collision algorithm based on tag estimation and slot location in this paper. The key technique can be divided into two parts: precisely tag estimation and slot location. Simulation and test results show that the proposed anti-collision algorithm performs better than the traditional algorithm despite its simplicity.
\end{abstract}

Keywords-Index Terms: Internet of Things, RFID, Anti-collision algorithms,slot location, tag estimation

\section{INTRODUCTION}

Radio frequency identification (RFID) is a contactless automatic identification technology, which automatically identifies the target by RF signals and gets the relevant data. It can complete information input and processing without human manage. Therefore, it's widely used in logistics, tracking, positioning and other fields [2] [3] [11].

RFID systems consist of reader, internet and tag. The reader is a powerful device with ample memory and computational resources. Tag stores object's information, it can be divided into two categories, such as passive tags which respond only at reader commands, active tags which have an on-board micro-controller, transceiver, memory, and power supply. Among tag types, the passive tags are emerging to be a popular choice due to their low cost [1] [4].In RFID systems, tags collisions happen when two or more tags share a common communication channel at the same time. It will result in the wastage of bandwidth, energy, and increases identification delays. The RFID anti-collision problem is one of the important and difficult problems in the RFID systems. Those methods which solve this problem are called anti-collision algorithm. It can be divided into two categories: binary algorithms based on tree structure and aloha algorithms based on probability distribution [2] [3] [7].

To date, some tag anti-collision algorithms have been proposed. In this paper, we mainly research the algorithm base on probability distribution. The pure ALOHA algorithm was applied in RFID systems in the early period, which is a simple multi-address communication approach[1] [2].This algorithm occurs a complete or partial collision [2] [3]. Tags collision is heavily when tags number is most. Therefore, the Slot ALOHA algorithm was proposed. Its collision occurs at slots boundary only, hence, it avoid partial collision. In Pure ALOHA (PA) and Slotted ALOHA (SA) algorithm, a tag with a high response rate will frequently collide with potentially valid responses from other tags. Therefore, Framed Slotted ALOHA (FSA) algorithms is proposed by selecting the frame size based on the number of tags and achieve the most system efficiency [2] [3]. Above all, slot location is neglected in traditional algorithm. Therefore, we present an improved anti-collision algorithm base on the estimation of tag and slot location in this paper. The simulation results show that the algorithm can improve the stability and the reading accuracy of the RFID systems.

This paper is organized as follows. In Section 2 the classic anti-collision is illustrated. Improved algorithm described in Section 3. The simulation results are presented in Section 4. Section 5 draws the conclusion.

\section{CLASSIC ANTI-COLLISION ALGORITHM}

Anti-collision algorithms are critical to the performance of RFID systems. Thus, they can be categorized into, space division multiple access (SDMA), frequency division multiple access (FDMA), code division multiple access (CDMA), and time division multiple access (TDMA).Briefly, SDMA algorithms [3] spatially separate the channel using directional antennas or multiple readers to identify tags. They, however, are expensive and require intricate antenna designs. On the other hand, FDMA [1] algorithms involve tags transmitting in one of several predefined frequency channels; thus, requiring a complex receiver at the reader. Lastly, systems based on CDMA [2] require tags to multiply their ID with a pseudo-random sequence before transmission. Unfortunately, CDMA based systems are expensive and power hungry.

In RFID systems, TDMA are the largest group of anti-collision algorithm. These algorithms can be classified as reader driven and tag driven. The former and latter are also called Reader-talk-first (RTF) and Tag-talk-first (TTF) respectively [1] [2]. Most applications use RTF algorithms, which can be further classified into Aloha and tree based algorithms. Note, there is also a hybrid class, which combines Aloha and tree algorithms. The basic idea behind RTF is that tags remain quiet until specifically addressed or commanded by a reader. On the other hand, TTF algorithms function asynchronously. This means a TTF tag announces itself to the reader by transmitting its ID in the presence of a reader. Tags driven algorithms are slow as compared to RTF algorithms.

In FSA algorithm, a frame is divided into several slots with same length. Tags randomly select a slot in the frame and begin to transmit information only at the start of the slot. Suppose the frame contains $\mathrm{N}$ slots and $\mathrm{n}$ tags are unidentified. Each tag randomly selects a slot of the frame with the probability, then we can deduce the probability that 
$\mathrm{k}$ tags simultaneous transmit information in one slot as follows [1]-[3] [7] [10].

$$
\text { - } P(k)=\left(\begin{array}{l}
n \\
k
\end{array}\right)\left(\frac{1}{N}\right)^{k}\left(1-\frac{1}{N}\right)^{n-k} \text {. }
$$

Accordingly, the expectation of the identified tags in the frame can be got.

$$
\text { - NP }(1)=\left(\begin{array}{l}
n \\
1
\end{array}\right)\left(\frac{1}{N}\right)^{1}\left(1-\frac{1}{N}\right)^{n-1} \cdot
$$

After an identified frame round, we count the number of empty slots, slots filled with one tag, and slots with collision as $\left\langle c_{0}, c_{1}, c_{k}\right\rangle$. S denotes the throughput. Now we compute the expectation of $\left\langle c_{0}, c_{1}, c_{k}\right\rangle$ and $\mathrm{S}$ as follows.

$$
\begin{gathered}
a_{0}{ }^{N, t}=N * P(1)=n\left(1-\frac{1}{N}\right)^{n-1} \\
a_{1}{ }^{N, t}=N * P(0)=N\left(1-\frac{1}{N}\right)^{n} \\
a_{k}{ }^{N, t}=N-a_{0}{ }^{N, t}-a_{1}{ }^{N, t} .
\end{gathered}
$$

E denotes the system efficiency.

$$
\begin{aligned}
& E=\frac{\text { tags (identified ) }}{N} . \\
& =\frac{c_{1}}{N}=\frac{n}{N}\left(1-\frac{1}{N}\right)^{n-1}
\end{aligned}
$$

The simulation result of system efficiency is shown in Fig. 1 , where $\mathrm{N}=256$.

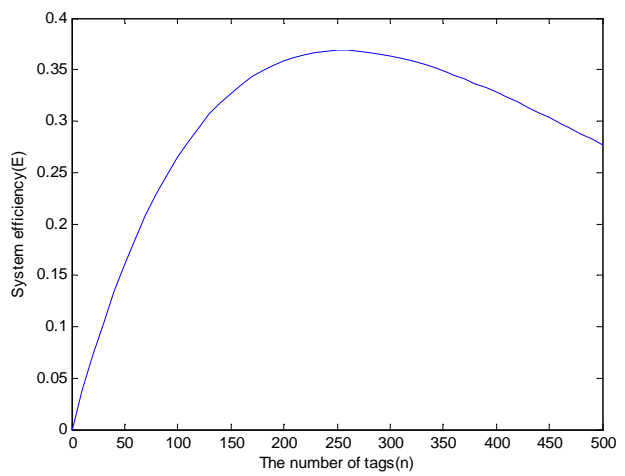

Fig.1. System Efficiency of FSA

Let the differentiation of (6) be zero

$$
\begin{aligned}
& \frac{d E}{d n}=\frac{1}{N}\left(1-\frac{1}{N}\right)^{n-1}+ \\
& \frac{n}{N}\left(1-\frac{1}{N}\right)^{n-1} \ln \left(1-\frac{1}{N}\right) \\
& =\frac{1}{N}\left(1-\frac{1}{N}\right)^{n-1}\left[1+n \ln \left(1-\frac{1}{N}\right)\right] \\
& =0
\end{aligned}
$$

Thus

$$
n=-\frac{1}{\ln \left(1-\frac{1}{N}\right)}
$$

$$
N=\frac{1}{1-e^{-\frac{1}{n}}}
$$

The equation (9) can be approximated by (10).

$$
\begin{aligned}
& N \approx \frac{1+1 / n}{1+1 / n-1} . \\
& =n+1, n>>1
\end{aligned}
$$

Above all, Fig.1 and theoretical results show that the system efficiency will get the maximum when the identification tags number equal to the frame length.

\section{THE PROPOSED ALGORITHM}

This anti-collision algorithm is applicable for large-scale RFID system. In real applications, the number of tags is unknown before identification. Therefore, the number of tags is estimated by using the statistical result of the number of empty slots, success slots and slots with collision after a read circle. Then according to the estimation result and the given frame length $(\mathrm{N})$, tags can be grouped. Therefore, in each circle, the reader can maximize the identification efficient. The tag is no longer grouped until the tag number is below a given value.

During the identification cycle, tags generate a rand number $M$, then it transfer $M$ to the reader when tag enter the zone of reader. Remainder which $\mathrm{M}$ divided by $\mathrm{N}$ is selected as slot; second, quotient which $\mathrm{M}$ divided by $\mathrm{N}$ is selected as translate sequence in the slot [4] [10].

\section{A. Tag Estimate}

In FSA algorithm, let $C=[\mathrm{c}(0) ; \mathrm{c}(1) ; \mathrm{c}(\mathrm{M}-1)]$ be $\mathrm{M}$ past observations of read result, empty slots, slots with collision and success slots number in a frame. Bayesian estimate considers number of tags as a random variable, not a deterministic one and $p(n \mid c)$ as $n$ 's the posterior distribution [8] [9].

When $C=\left\langle c_{0}, C_{1}, c_{k}\right\rangle$, the posterior distribution of tag can be got.

$$
p(n \mid c)=\frac{N !}{c_{0} ! c_{1} ! c_{k} !} P(0)^{c_{0}} P(1)^{c_{1}} P(k)^{c_{k}}
$$

The tag quantity of Bayesian estimate can be shown as

$$
\bar{n}=\arg \min _{\tilde{n} \in \Omega} \sum_{n=1}^{\infty} J(\tilde{n}, n) p(n \mid c)
$$

$J(\tilde{n}, n)$ is the cost function; $p(n \mid c)$ is $n$ 's the posterior distribution.

We assume sample space of the number of tags as follows.

$$
\Omega=\left\{\tilde{n} \mid c_{1}+2 c_{k} \leq \tilde{n} \leq N\right\}
$$

Let $J(\bar{n}, n)=(\tilde{n}-n)^{2}$ estimation tag number can be expressed as.

When $n \rightarrow \infty$, the Taylor series of (8) is shown in (9). 


$$
\bar{n}=\sum_{n=1}^{N} n \bar{p}(n \mid c)
$$

Where we let $p$ be normalized $\bar{p}$ which can make

$$
\sum_{n=1}^{N} \bar{p}(n \mid c)=1
$$

\section{B. Tag Identification}

Let $\mathrm{n}$ be tag number, $T_{0}$ be time which start instruction deliver, $T_{1}$ be time which "silence" instruction transmission deliver, $T_{2}$ be slot length, $\mathrm{S}$ be transmit slot in the cycle, $\mathrm{Q}$

be transmit sequence in this slot, then we can get:

$$
\begin{array}{r}
S=M \% N \text {. (15) } \\
Q=M / N . .
\end{array}
$$

The system efficiency of the proposed algorithm as follows.

$$
\text { - } E=\frac{\bar{n} T}{T_{0}+N T_{2}+\bar{n} T+\bar{n} T_{1}}
$$

\section{Simulation Results}

We consider a reader and the large-scale tags under error-free channel environment. In the proposed method, let $\mathrm{T}=0.1 \mathrm{~s}, T_{0}=0.005 \mathrm{~s}, T_{2}=0.007 \mathrm{~s}, T_{1}=0.005 \mathrm{~s}$. To evaluate the system performance, we compare the system efficiency of the proposed algorithm with traditional FSA, and set the frame size from 64 to 256 and vary the number of tags from 0 to 500 .

As shown in Fig.2, the simulation results of system efficiency of traditional FSA and the proposed algorithm in the same environment. Form Fig.2, we can see that the system efficiency of the proposed algorithm is higher than that of traditional FSA in the same frame and number of tags. The system efficiency of the proposed algorithm get a relative stable result when the number of tags is greater than $\mathrm{N}$ in the proposed algorithm. However, the system efficiency of traditional FSA is gradually slow down when the number of tags is greater than $\mathrm{N}$, and gradually close to zero when the number of tags is more greater than $\mathrm{N}$. The system efficiency can enhance $50 \%$ at least in the same $\mathrm{N}$, and the difference of the system efficiency between traditional FSA and the proposed algorithm is increase with the increasing of the number of tags.

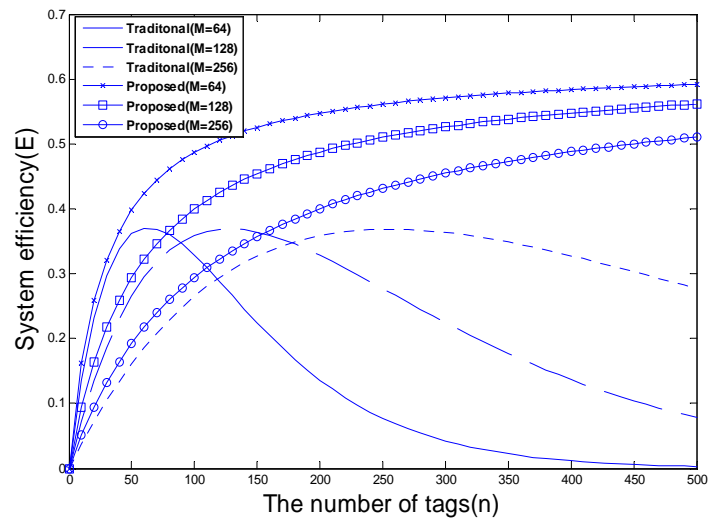

Fig. 2. Simulation results of system efficiency of traditional FSA and the proposed algorithm

The paper proposed the proposed algorithm based on slot location and tag estimation. The simulation result show the system efficiency of the proposed algorithm is stable in specific condition. The reason is that we grouped tag when the number of tags is greater than $\mathrm{N}$ and the tag identify by slot location.

\section{CONCLUSION}

To achieve the optimal performance of RFID systems, we have proposed an efficient method in this paper. We explored the benefits of the precisely estimation of tag quantity and identification base on slot location. The proposed method can improve the performance of system compared with that of the traditional method.

\section{ACKNOWLEDGMENT}

This work is fully supported by Sichuan Province Science Foundation for Youths [No. 2011JQ0034], and Science Founding of Artificial Intelligence of key laboratory of Sichuan Province [Project No. 2012RYJ05], and Science Founding of Sichuan University of Science \& Engineering [Project No. 2011KY10].

\section{REFERENCES}

[1] K.Finkenzeller, "RFID Handbook, Fundamentals and Applications in Contactless Smart Cards and Identification.” John Wiley and Sons Ltd,2003.

[2] L.A.Burdet, "RFID multiple access methods." Technical Report.

[3] K. Finkenzeller and R. Waddington, "RFID Handbook: Radio-Frequency Identification Fundamentals and Applications." John Wiley \& Sons, Jan.2000.

[4] H. Vogt, "Efficient object identification with passive RFID tags." in IEEE PerCom, (TX, USA), 2002.

[5] Chen-Chung Liu,'Tag Quantity Estimation for RFID Based on Probability." 2010 International Symposium on Computer, Communication, Control and Automation.

[6] Yao-feng Xue, ,'RFID Dynamic Grouping Anti-collision Algorithm Based on FCM." 2009 International Joint Conference on Bioinformatics, Systems Biology and Intelligent Computing.

[7] Chen W T, "An accurate tag estimate method for improving the performance of an RFID anti-collision algorithm based on dynamic frame length ALOHA." IEEE Transactions on Automation Science and Engineering, 2009, 6(1): $9 ; 15$ 
[8] Wu Hai-Feng, Zeng Yu "Tag Estimate and Fame Length for Dynamic Frame Slotted ALOHA Anti-collision RFID System." ACTA AUTOMATICA SINICA,2010

[9] H. Vogt, "Multiple object identification with passive RFID tags." 2002 IEEE international conference on systems, man and cybernetics, 2002, (3), pp. 651-656.

[10] T. W. Hwang, B. G. Lee, Y. S. Kim, et al, "Improved anti-collision scheme for high speed identification in RFID system." Proceedings of the first international conference on innovative computing, information and control (ICICIC'06),2006, pp. 49-452.

[11] SUN Wensheng, LIU Xianbao, "Research and improvement on RFID system tags anti-collision." College of Communication Engineering, Hangzhou Dianzi University, Hangzhou 310018, China 